\title{
Evidence for photochemical transformations in porous silicon
}

\author{
V. B. Shevchenko, V. A. Makara, O. V. Vakulenko, O. I. Dacenko, O. V. Rudenko \\ Taras Shevchenko Kyiv Univ., 6, prospect Glushkova, 252127 Kyiv, Ukraine, \\ tel. 38044 5134058, fax: 38044 2662326, e-mail: doce@hq.ups.kiev.ua
}

\begin{abstract}
The dynamics of the variation of photoluminescence intensity (PLI) of porous silicon (PS) samples subjected to laser irradiation $(337 \mathrm{~nm}, 3.7 \mathrm{~mW})$ during their ageing in air has been studied. The PLI turned out to increase rapidly under UV irradiation and to flatten out in several hours. The subsequent irradiation leads to intensity degradation, which may be explained by the luminescence fatigue effect. At the same time, the PLI of the unilluminated sample almost does not change during the experiment. It turned out that the anomaly as a small surge down is observed on the PLI evolution curve at the stage of the initial monotonous increase of PLI after a short-time (1 to 2 minutes) interruption of the laser illumination of the sample, whereas this anomaly is a surge up at the stage of the monotonous fall of the PLI curve. In the case of a long-time (tens of hours) discontinuation of illumination, the anomaly was similar for all the portions of the PLI curve. The described results are explained by effect of two competing factors which are the luminescence fatigue and light-induced formation of unstable (molecular) chemical bonds that can transform to the stable atomic ones.
\end{abstract}

Keywords: porous silicon, nanostructure, laser irradiation, luminescence fatigue.

Paper received 21.06.99; revised manuscript received 29.06.99; accepted for publication 12.07.99.

\section{Introduction}

Porous silicon (PS) has been known to researchers for a rather long time. The interest in it has sharply risen in 1990 due to the discovery of its ability to emit an intense visible luminescence [1]. However, the nature of the PS red luminescence has not been exactly established despite the intensive investigations.

The extreme sensitivity of luminescence to ambient is one of the most distinguished properties of PS. On the one hand, such a property complicates the practical use of PS in technology due to its instability in the air [2-6]. On the other hand, this property allows one to control the PS luminescent parameters $[7,8]$. Studies in this direction can also be useful for elucidation of the luminescence mechanism.

This work is devoted to the study of PS photoluminescence (PL) intensity evolution in the air under UV laser irradiation.

\section{Experiment}

The PS layers were obtained by electrochemical anodizing of (111) $p$-Si wafers in the $1: 1$ mixture of $48 \% \mathrm{HF}$ and isopropyl alcohol at a current density of $10 \mathrm{~mA} / \mathrm{cm}^{2}$ for 5 min. As-prepared PS displayed a weak PL signal comparable to the noise level of the experimental setup. After anodizing, the samples were kept in the air for 3 months. Their luminescence intensities essentially increased during that period of time, which occurred, probably, due to effects of porous layer oxidation $[9,10]$. Immediately before the experiment, the samples were chemically etched in HF for 3 seconds. Such a treatment returned the nanostructure surface to the initial state, that is, removed the surface oxide and saturated the external silicon bonds by hydrogen. Furthermore, the porous layer surface cracks as a result of the HF treatment, which promotes the penetration of oxygen into the depth of porous layer and accelerates the PS ageing process $[10,11]$. 


\section{B. Shevchenko et al.: Evidence for photochemical transformations...}

The integral intensity of PL was measured at room temperature in the visible, the luminescence was excited by unfocused beam of ILGI-503 laser $(337 \mathrm{~nm}, 3.7 \mathrm{~mW})$. The beam diameter was close to $2.5 \mathrm{~mm}$ in the sample plane. A photodiode with spectral sensitivity in the range of 365 to $750 \mathrm{~nm}$ was used as a light detector. The UV excitation light scattered from the sample was cut off by the GS-4 absorptive filter placed before the emission detector.

During the experiments for the study of the laser emission effect on the PS PL, the laser was periodically switched on for a few hours during a week. All the rest time it was switched off. The duration of the laser continuous operation did not exceed 4 to 5 hours, after which the sample was not illuminated for tens of hours. During the laser operation, the beam was intercepted by a screen several times. By this means we studied the effect of the UV irradiation on the sample PL and also observed the effect of a short- and long-time interruption of irradiation action on the evolution of PL intensity.

For comparison, we used a sample which was stored in the dark and illuminated by laser only episodically during the scheduled measurements of its PL intensity.

\section{Results and discussion}

The results of the study of the PL intensity of the sample irradiated by the laser are shown in Fig.1. The time when the laser was switched on is plotted along the $\mathrm{X}$-axis. The breaks in the laser irradiation of the sample are marked by the arrows, their durations are indicated.

As it is shown in Fig.1, the chemical treatment of samples in concentrated HF at the beginning of experiment has led to PL intensity increase by a factor of 2 . At the surface of silicon nanostructure, dissolution of the oxygencontaining compounds of Si formed in anodizing and storing the prepared PS in the air, evidently occurs during such a treatment. These compounds are the PL sensitizers in PS $[12,13]$. According to the supposition of [10], the interaction of PS with oxygen takes place preferentially near the surface of porous layer when storing the sample in the air. After a prolonged contact with the atmosphere, an emitting layer is formed in the near-surface region. This layer is $\mathrm{SiO}_{\mathrm{x}}$ sensitizer with embedded Si nanocrystals, in which the radiative recombination occurs. At the sample surface, however, a rather thick oxide layer, where the complete oxidation of the nanostructure has happened, i.e. oxide layer without residuals of nanocrystalline silicon can be formed under certain conditions. This layer would not participate in the luminescence. On the contrary, its presence would negatively affect the quantum yield of the sample because the layer absorbs the excitation light. Therefore, its removal by a short-time treatment in HF would increase the intensity of sample emission.

Not taking into account the short-time intensity drop for the first minutes of the experiment (Fig.1) and the meaningful anomalies related to breaks of laser beam action, one can conclude that the luminescence quantum yield monotonously rises and flattens out during the experiment. In principle, this correlates with the most of data for the PL intensity behaviour when ageing a PS sample in the air (see, e.g., [3,4,6,9,11]). The causes for the rise of PS PL intensity in the air are associated with the atmospheric oxidation of the nanostructure [10]: increase of the sensitizer mass (which is the Si oxide for UV region of excitation [12]), effective saturation of the silicon dangling bonds (nonradiative recombination cen-

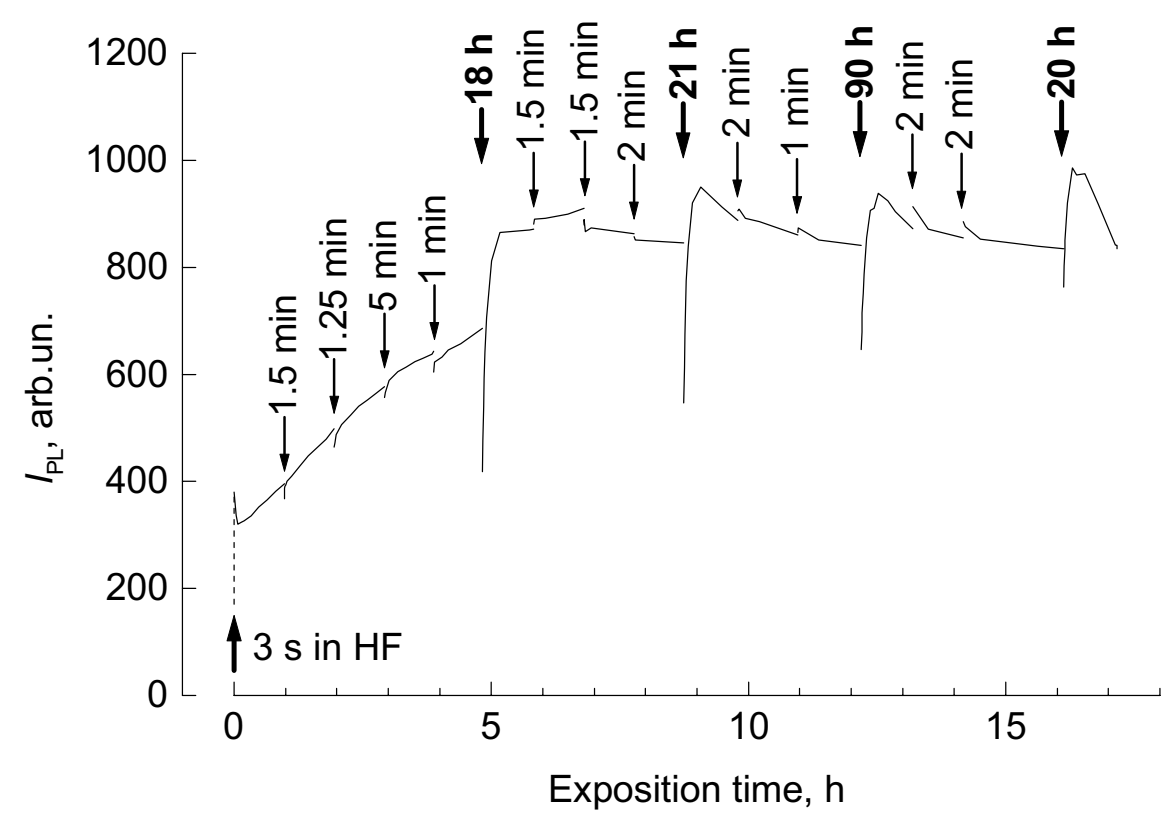

Fig. 1. Evolution of PL intensity of the PS sample exposed to the air under the laser illumination. Breaks in irradiating the sample are marked as the jumps on the curve, their durations are indicated nearby. 


\section{B. Shevchenko et al.: Evidence for photochemical transformations...}

ters [2]) by oxygen, and the nanostructure modification due to the oxidation.

For the reference sample which was stored in the dark, a monotonous increase of PL intensity is documented as well, but it was significantly slower than that for the laser-illuminated sample. This indicates the effect of laser irradiation on the processes happening in porous layer.

It should be noted that the pattern of PL intensity evolution in Fig. 1 is somewhat distorted due to the luminescence fatigue effect $[14,15]$. The presence of this phenomenon manifests itself most evidently at the final stage of our experiments (the portion of flattened intensity in the curve). At the stage of continuous illumination of the sample, the PL intensity decreases monotonously rather than reaches a steady-state value, which would be expected in the case of episodic illumination of the sample by excitation light while it is exposed to the air [11]. This effect is obscured on the background of the total rise of intensity at the outset of experiment.

The luminescence fatigue should apparently be considered as a reason of the fast drop of the intensity for the first minutes of experiment (Fig.1). However, this is unlikely to be the main reason because we observed a similar intensity drop for as-prepared samples and the samples as-etched in HF without continuous laser illumination. The authors of Ref. [16] also observed the fast degradation of PS PL at the outset of experiment when exposing the samples in the pure oxygen atmosphere. They concluded that the intensity decrease is conditioned by formation of Si dangling bonds at the PS surface. Taking into account the suggestion that the hydrogen coating of the nanostructure is changed by oxygen in as-prepared or HF-etched sample as a reason of increase of the PL intensity of aged PS $[12,17]$, one can conclude that the hydrogen escape from PS surface runs more rapidly than the surface saturation by oxygen. The PL intensity decreases as a result of the temporary formation of $\mathrm{Si}$ dangling bonds being the centers of nonradiative recombination [2].

The anomalies related to temporal break of irradiation for the short ( 1 to 5 minutes) and long (tens of hours) terms attract the greatest interest on the PL evolution curve (Fig.1).
The long-term break of sample illumination by the laser light leads to significant drop of PL intensity. As a result of the further irradiation of sample, the complete restoration of the PL intensity occurs for the first 20 minutes, the magnitude of the shading-induced intensity drop relative to that of the intensity measured before switching off the illumination constantly decreasing. The noted anomalies related to the illumination interruption are not observed at all for stable samples stored in the air under illumination for a long time.

The PL intensity jumps as a consequence of temporary interruption of laser irradiation of the PS may be explained by formation of unstable molecular bonds on the Si surface of nanocrystallites with air atomic groups during photochemical transformations, the silicon skeleton surface turns out to be passivated due to this.

An illumination interruption causes the reverse transformations at the nanostructure surface, i.e. decaying the unstable passivating coating. As a result, the sample PL quantum yield after repeated switching on the light turned out to be lower than that before the shading, although the intensity rapidly restores under light due to saturation of dangling bonds. One can see that not only does the intensity rapidly restore (Fig.1), but it also exceeds the magnitude before the shading. In our opinion, the PL fatigue effect manifests itself here. Due to this, the intensity magnitude before switching off the light is lower than that after several hours of the sample being in the dark.

The stability of the surface coating evidently rises irreversibly with time. This is confirmed by absence of the anomalies related to the illumination termination in stable samples being in the air for a long time.

The effect of a short-time interception of the laser beam on the PL intensity of the irradiated sample is given in Fig.2 in more detail. The time intervals while the laser beam did not impinge on the sample surface are marked by the dashed line. Fig.2a illustrates the case of the laser beam interception at the stage of the total rise of PL quantum yield of the sample. Fig. $2 b$ shows the laser interruption effect at the stage of the flattened curve of the PL intensity evolution or, more precisely, during fatigue-induced PL efficiency degradation
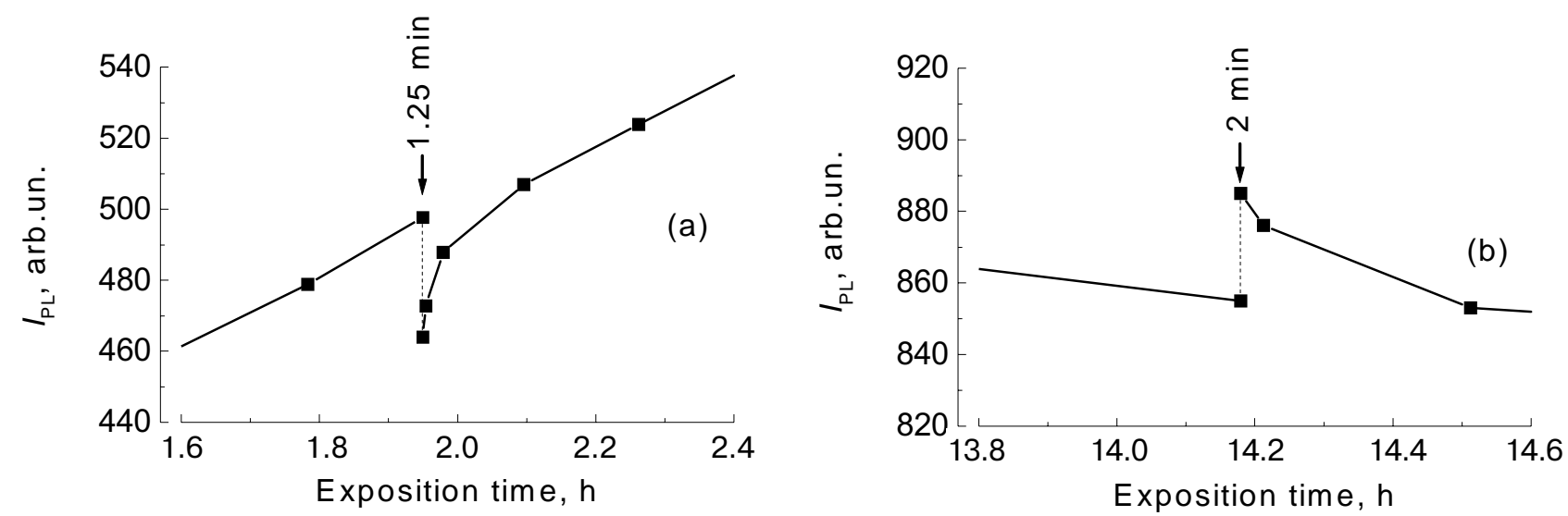

Fig. 2. Behaviour of PS sample PL intensity in short-time interception of laser irradiation at the stage of the PL efficiency total rise (a) and in the flattened part of the plot (b). 


\section{B. Shevchenko et al.: Evidence for photochemical transformations...}

while continuous irradiation. In the former case (Fig.2a), the PL intensity just after restoration of the laser action turns out to be lower that that before the laser beam interception; the PL efficiency of the sample increases for the first minutes of illumination. In the latter case (Fig.2b), the PL intensity of sample noticeably increases while the laser beam does not illuminate the sample surface. It decreases in the course of the further illumination.

The reasons of the PL intensity jump down (Fig.2a) after the short-time interception of laser beam are obviously the same as in the case of the long-time switching the light off (Fig.1). The instability level of the surface silicon compounds is the highest at the first stage of nanostructure surface formation (saturation of the dangling bonds) and, therefore, destruction of the unstable bonds would be essential even during the short-time illumination interruption and, as a result, degradation of the PL yield would be observed. The surface coating of nanostructure becomes more stable with time, hence degradation of the PL efficiency is not observed after the short-time shading of the sample (Fig.2b). At this stage, another effect affects the PL intensity instead. This is the luminescence fatigue. Therefore, just after illumination restoration, the intensity has a higher value than that before shading.

\section{Conclusions}

The luminescence efficiency of PS sample rises monotonously when exposing the sample to the air and flattens out some time after. The increase of PL quantum yield is significantly accelerated while illuminating the sample by UV laser radiation. As a result of restoration of sample illumination after its temporary interruption, the PL intensity value appears to be unequal to that after the interception of laser beam. According to this supposition, two competing factors affect the PL intensity behaviour: 1) luminescence fatigue and 2) light-induced formation of unstable (molecular) chemical bonds, which can transform to the stable ones with time.

\section{References}

1. L. T. Canham. Silicon quantum wire array fabrication by electrochemical and chemical dissolution of wafers // Appl. Phys. Lett. 57, p. 1046-1048 (1990).

2. M. A. Tischler, R. T. Collins, J. H. Stathis, and J. C. Tsang. Luminescence degradation in porous silicon // Appl. Phys. Lett. 60, p. 639-641 (1992).
3. T. Maruyama and S. Ohtani. Photoluminescence of porous silicon exposed to ambient air // Appl. Phys. Lett. 65, p. 1346-1348 (1994).

4. C.-H. Lin, S.-C. Lee, and Y.-F. Chen. Morfologies and photoluminescence of porous silicon under different etching and oxidation conditions // J. Appl. Phys. 75, p. $7728-7736$ (1994).

5. R. Sabet-Dariani, N. S. McAlpine, and D. Haneman. Electroluminescence in porous silicon // J. Appl. Phys. 75, p. 8008-8011 (1994).

6. S. Banerjee, K. L. Narasimhan, and A. Sardesai. Role of hydrogen- and oxygen-terminated surfaces in the luminescence of porous silicon // Phys. Rev. B. 49, p 2915-2918 (1994).

7. A. Bsiesy, J. C. Vial, F. Gaspard, R. Herino, V. Ligeon, F. Muller, R. Romestain, A. Wasiela, A. Halimaoui, and G. Bomchil. Photoluminescence of high porosity and of electrochemically oxidized porous silicon layers // Surf. Sci. 254, p. 195-200 (1991).

8. V. P. Bondarenko, A. M. Dorofeev, V. I. Levchenko, A. I. Lukomskiy, L. I. Postnova. Method of control of porous silicon luminescence parameters in the visible // Pisjma v Zhurn. Tekh. Fiz. (Russ. Tech. Phys. Lett.) 20, p. 61-65 (1994).

9. V. A. Makara, V. A. Odarych, O. V. Vakulenko, and O. I. Dacenko. Ellipsometric studies of porous silicon // Thin Solid Films 342 , p. 230-237 (1999).

10. O. I. Dacenko, V. A. Makara, S. M. Naumenko, T. V. Ostapchuk, O. V. Rudenko, V. B. Shevchenko, O. V. Vakulenko, and M. S. Boltovets. Evolution of the porous silicon sample properties in the atmospheric ambient // J. Luminescence 81, p. 263-270 (1999).

11. V. A. Makara, M. S. Boltovets, O. V. Vakulenko, O. I. Dacenko, S. M. Naumenko, T. V. Ostapchuk, and O. V. Rudenko. Peculiarities of porous silicon photoluminescence after chemical etching in HF // Zurn. Prikl. Spetrosc. (Belorus. J. Appl. Spectrosc.) 66, p. 423-427 (1999).

12. I. A. Buyanova, I. Ya. Gorodetsky, N. E. Korsunskaya, T. N. Mel'nik, I. M. Rarenko, A. U. Savchuk, and M. K. Sheynkman. Sensitized luminescence of porous silicon and its polarization characteristics // Fiz. Tekh. Polupr. (Russ. Phys. and Tech. of Semicond.) 30, p. 1516-1524 (1996).

13. V. A. Makara, O. V. Vakulenko, O. I. Dacenko, V. M. Kravchenko, T. V. Ostapchuk, O. V. Rudenko, M. S. Boltovets, V. O. Fesunenko. Effect of boron diffusion doping of silicon on the micromechanical and luminescence properties of porous layers // Thin Solid Films 312, p. 202-206 (1998).

14. I. M. Chang, S. C. Pan, and Y. F. Chen. Light-induced degradation on porous silicon, Phys.Rev.B. 48, p. 8747-8750 (1993).

15. K. S. Zhuravlev, N. P. Stepina, T. S. Shamirzaev, E. Yu. Buchin, N. E. Mokrous. Decay and rise kinetics of porous silicon photoluminescence under continuous laser radiation // Fiz. Tekh. Polupr. (Russ. Phys. and Tech. of Semicond.) 28, p. $482-487$ (1994).

16. P. K. Kashkarov, E. A. Konstantinova, and V. Yu. Timoshenko. Mechanisms of molecule adsorbtion effect on the recombination processes in porous silicon // Fiz. Tekh. Polupr. (Russ. Phys. and Tech. of Semicond.) 30, p. 1479-1489 (1996).

17. V. G. Golubev, A. V. Zherzdev, G. K. Moroz, A. V. Patsekin, and D. T. Yan. Strong photoinduced increase of photoluminescence intensity of anodically oxidized porous silicon // Fiz. Tekh. Polupr. (Russ. Phys. and Tech. of Semicond.) 30, p. 852-863 (1996). 